

\section{DISCLAIMER}

This report was prepared as an account of work sponsored by an agency of the United States Government. Neither the United States Government nor any agency Thereof, nor any of their employees, makes any warranty, express or implied, or assumes any legal liability or responsibility for the accuracy, completeness, or usefulness of any information, apparatus, product, or process disclosed, or represents that its use would not infringe privately owned rights. Reference herein to any specific commercial product, process, or service by trade name, trademark, manufacturer, or otherwise does not necessarily constitute or imply its endorsement, recommendation, or favoring by the United States Government or any agency thereof. The views and opinions of authors expressed herein do not necessarily state or reflect those of the United States Government or any agency thereof. 


\section{DISCLAIMER}

Portions of this document may be illegible in electronic image products. Images are produced from the best available original document. 


\title{
ANGULAR DISTRIBUTIONS OF ELECTRONS EJECTED BY FAST PROTONS*
}

\author{
L. H. Toburen \\ Battelle Memorial Institute \\ Pacific Northwest Laboratory \\ Richland, Washington 99352
}

Measurements of the energy and angular distributions of electrons ejected in ion-molecule collisions have been shown to be a sensitive test of theoretical descriptions of the ionization process. The measurements of Kuyatt and Jorgensen, (1) Rudd and Jorgensen, (2) and Rudd, Sautter and Bailey (3) for protons on hydrogen and helium (proton energies from 0.05 to $0.3 \mathrm{MeV}$ ) have, when compared to theory, illustrated the inadequacies of the Born approximation and binary encounter theory in describing details of the ionization process. Discrepancies between theory and measurements, such as were observed in the angular distributions of electrons, have lead to further developments in the theory of ionization. In general, however, these theoretical treatments involve approximations which restrict the range of validity of the results. In his recent work, for instance, Macek cautions that for $0.3 \mathrm{MeV}$ proton impact, "a first order approximation generally does not adequately describe the detailed distribution of electrons in energy and angle." (4) This inadequacy of first order approximations may be expected since $0.3 \mathrm{MeV}$ protons are only moving with a velocity 3.5 times the velocity of an electron in the first Bohr orbit

\footnotetext{
*This paper is based on work performed under United States Atomic Energy Commission Contract AT(45-1)-1830.
} 
of the hydrogen atom. The results described in the present paper are an extension of the measurements of doubly differential cross sections to proton energies of $2.0 \mathrm{MeV}$ for helium targets and $1.5 \mathrm{MeV}$ for molecular hydrogen targets. It is expected that these results will more adequately represent the energy range where theoretical calculations which utilize first order approximations may be applied.

A schematic drawing of the experimental arrangement is shown in Fig. 1. This apparatus as well as the measurement technique has been described previously ${ }^{(5)}$ and will be only briefly discussed here. A proton beam from a Van de Graaff generator was energy analyzed, collimated, and passed through a differentially pumped target cell. Electrons ejected from the target. gas were energy analyzed by a clindrical mirror electrostatic analyzer with an angular acceptance of approximately 5 degrees. The electron energy spectra were obtained at angles from 20 to 130 degrees with respect to the forward direction of the proton beam.

The general features of the angular distributions of ejected electrons are illustrated in Fig. 2 by a comparison of our results for $2.0 \mathrm{MeV}$ proton impact on helium to the theoretical results of Bonsen and Vriens ${ }^{(6)}$ which are based on binary encounter theory. Their calculation includes the effects of the velocity distribution of the bound electrons but does not include such features as the distortion of the electron distribution by the passing proton or the influence of the long-range interaction between the ejected electron and the outgoing proton. The agreement between our measurements and the results of binary encounter theory, shown in Fig. 2, is excellent for intermediate angles where one 
would expect this semi-classical theory to be most applicable. The discrepancies observed for large emission angles have been attributed to the interaction of the outgoing electron with the rest of the atom in which the electron distribution has been distorted by the interaction of the passing proton. The effects of this interaction are observed for lowenergy electrons and seem to be negligible for electrons with energies greater than a few hundred electron volts. The enhancement of the cross section for small angles has been the topic of much discussion and has been attributed to the long-range interaction between the ejected electron and outgoing proton.

The erhancement of the emission cross sections for small angles has been the subject of calculations by Salin! (7) and Macek. (4) Salin used a velocity depencent effective charge and the Born approximation, whereas, Macek's calculation was based on Faddeev's equations. Both approaches show qualitative agreement with the work of Rudd, et al., (3) however, quantitative agreement could not be obtained for proton energies as low as those used by Rudd, et al. Macek has recently extended his calculations to higher proton energies and a comparison of our measurement cross sections to his results is shown in Fig. 3 for $1.0 \mathrm{MeV}$ protons on molecular hydrogen. The Born cross sections show in Fig. 3 (and subsequent figures) were also calculaied by Macek. (8) The cross sections calculated by Macek from the Faddeev equations are restricted to ejected electron energies greater than several Rydbergs by approximations used in his development of the theory. Therefore, the discrepancies indicated in Fig. 3 between the measured and calculated cross sections for the ejection of $20 \mathrm{eV}$ electrons are not 
unexpected. As the ejected electron energy increases, agreement between our measurements and Macek's results improves with close agreement being obtained for electron energies near $1000 \mathrm{eV}$. On the other hand, cross sections calculated for small angles using the Born approximation, appear to be nearly a constant factor smaller than measured values with no improvement in the agreement as the electron energy increases. The largest discrepancy between the measured cross sections and those calculated using the Born approximation occurs at small angles for electron energies near 544 $\mathrm{eV}$. The underestimation of the small angle cross sections by the Born approximation is associated with the long-range proton-electron interation which is strongest when the incident proton and ejected electron have the same velocity, a condition which is met by $1.0 \mathrm{MeV}$ protons and $544 \mathrm{eV}$ ejected electrons. For electron energies greater than $100 \mathrm{eV}$ and emission angles larger than approximately 50 degrees, cross sections based on the Born approximation and on Macek's development of the Faddeev equations are in good agreement with each other and with measured values. First order approximations such as the Born calculation and the Macek's theory are expected to provide reliable estimates of the collision cross sections for high energy protons. In Fig. 4 are shown the results of measured and calculated cross sections for ejection of electrons with velocities equal to the incident proton energy for the range of proton energies studies in our work $(0.3$ to $1.5 \mathrm{MeV})$. The measurements of Rudd, et al. (3) for $0.3 \mathrm{MeV}$ proton impact are included in order to indicate the close agreement between the independent measurements. As the proton 
energy increases, the results of the liacek theory approach the experimental values with close agreement observed for $1.5 \mathrm{MeV}$ proton impact. Both the measured cross sections and those calculated by Macek tend toward the Born cross section as the proton energy increases; however, the effect of the long-range electron-proton interaction is still very much in evidence for proton energies as high as $1.5 \mathrm{MeV}$.

From a' comparison of experimental and theoretical cross sections for protons on hydrogen, it appears that the Macek theory provides reliable angular distributions for emission angles as small as 20 degrees for 1.5 $\mathrm{MeV}$ impact and angles to 30 degrees for $1.0 \mathrm{MeV}$ protons. Unfortunately, our experimental arrangement does not allow us to obtain results for emission angles smaller than 20 degrees. With the close agreement between our measured cross sections and those calculated by Wacek for fast protons on hydrogen, we were somethat surprised at the discrepancies which occur between measured and calculated cross sections for helium. In Fig. 5, we compare our measurements with results of the Macek theory for $1.0 \mathrm{MeV}$ proton on helium and electron energies of 500,544 , and $600 \mathrm{eV}$. We have a.lso measured electron emission cross sections for $2.0 \mathrm{MeV}$ deuterium ions on helium and these results are shown in Fig. 5 along with the proton data. A $2.0 \mathrm{MeV}$ deuterium ion and a $1.0 \mathrm{MeV}$ proton have the same velocity, therefore, the precise agreement in the electron emission cross sections for these two projectiles is to be expected. The result which we did not expect was that the calculated cross sections for electron emission from helium were approximately 30 percent smaller than the measured values for emission angles from 20 to 40 degrees. This disagreement is in contrast to the results for 


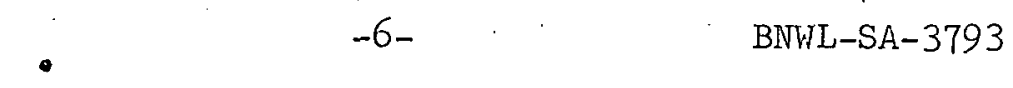

hydrogen where agreement was excellent for angles greater than approximately 30 degrees and where, when discrepancies did exist, they were such that the calculated values vere larger than measured ones. This underestimation of the small angle cross sections by the Macek theory was also unexpected in light of previous work for protons on helium at low energies (4) where the theoretical. cross sections were always larger than measured values.

One may speculate as to the processes which lead to electron emission in order to estimate to some extent what effect the change in target gas may have on the angular dependence of the emission cross sections. Clearly, the peak in the angular distributions which occurs near 60 degrees is due to a direct interaction between the proton on a loosely bound electron. The magnitude of this "binary encounter peak" depends roughly on the number of electrons in the outer electronic shell of the atom or molecule., Molecular hydrogen and helium, both have two loosely. bound electrons and, as expected, the magnitude of the peak is nearly the same for each gas. This peak is well represented by the semi-classical binary encounter calculations as well as the Born approximation and Macek theory. On the other hand, the enhancement of the cross section for small angle emission is not accounted for in either the plane wave Born approximation or binary encounter theory. - The theory of Macek, which does account for an' enhancement of the small angle emission cross sections, does so by including an interaction between the ejected electron and the outgoing proton. The effect of this interaction is that the proton pulls the electron along and, as Macek has commented, the component of the electron spectrum due to this process could be termed "an extrapolation in energy of charge exchange to an excited state of hydrogen." 
Crooks and Rudd ${ }^{(9)}$ have investigated this contribution to the electron spectrum by observing the peak which Macek predicts at an emission angle of zero degrees and find that the magnitude of the peak varies with proton energy in accordance with the charge transfer cross sections. Therefore, one may expect that the magnitude of the small angle electron ejection cross sections may show a dependence on target gas similar to that exhibited by the electron-capture charge-transfer cross sections.

In Fig. 6 are shown the results of our measurements for $1.0 \mathrm{MeV}$ protons on several gas targets where the cross sections considered are for an electron energy corresponding to the ejected electron and outgoing proton having the same velocity. The ratio of the cross sections for electron ejection al 60 degrees for the different target gases is nearly the same as the ratio of the number of loosely bound electrons for the corresponding target gases. This correlation would be expected in the binary encounter region of the angular distribution since the direct interactions will occur predominantly with the loosely bound electrons. At: smaller emission angles, the cross sections exhibit a considerably different dependence on the target material; this is most obvious for the case of hydrogen and helium. Electron capture cross sections exhibit a similar variation in magnitude as a function of the different target molecules. If one were to extrapolate our measurements for nitrogen to zero emission angle and normalize the relative magnitude of the charge transfer cross sections at that point, then the arrows shown in Fig. 6 indicate the relative magnitudes of the electron capture cross sections for the different targets $(10)$ studied (charge transfer cross sections for $1.0 \mathrm{MeV}$ protons on $\mathrm{Xe}$ are not available). These 
relative cross sections are very similar to the electron emission cross sections as the emission angle approaches zero which tends to support the continuum state charge transfer mechanism. The ratio of the cross sections for hydrogen and helium can also be obtained from the calculations of Macek. His calculations provide helium cross sections which are approximately a factor of 3 greater than molecular hydrogen for angles of 40 degrees and smaller. These results compare to our measurements in which the ratio of helium to hydrogen cross sections varies from approximately 3 at 40 degrees to 6 at 20 degrees and the ratio deduced from charge transfer measurements, which one might consider applicable at zero degrees, is . approximately 9. It should also be noted that, as the target molecule increases in complexity, the enhancement of the cross section in the forward direction increases and the minimum which occurs for small angles moves toward slightly larger angles. Whereas we could not observe a definite upturn in the hydrogen or helium cross sections for angles as small as 15 degrees, the emission cross sections for xenon begin to increase for angles smaller than 40 degrees. 


\section{FIGURE CAPTIONS}

Fig. I A schematic drawing of the apparatus.

Fig. 2 Angular distributions for electrons of selected energies ejected from helium by $2.0 \mathrm{MeV}$ protons. Theoretical results (a) are from Ref. 6.

Fig. 3 Angular distributions for electrons of selected energies ejected from molecular hydrogen by $1.0 \mathrm{MeV}$ protons. Theoretical results (a) see $\operatorname{Ref} .4$ and (b) see Ref. 8 .

Fig. 4 Angular distributions of electrons ejected with velocities equal to the incident proton velocity. Previous measurements: (a) see Ref. 3. Theoretical resulls: (b) see Ref. 4, (b) see Ret: 8.

Fig. 5 Angular distributions for selected electron energies for ejection of electrons from helium by $1.0 \mathrm{MeV}$ protons. Theoretical results: (a) see Ref. 4, (b) see Ref. 8.

Fig. 6 Angular distributions for ejection of $544 \mathrm{eV}$ electrons from several gas targets by $1.0 \mathrm{MeV}$ protons. The arrows represent the relative magnitude of the electron capture charge transfer cross sections of Ref. 10 . 


\section{REFERENCES}

1. C. E. Kuyatt and T. Jorgensen, Jr., Phys. REv. 130, 1444 (1963).

2. M. E. Rudd and T. Jorgenson, Jr., Phys. Rev. 131, 666 (1963).

3. M. E. Rudd, C. A. Sautter and C. L. Bailey, Phys. Rev. 15I, 20 (1966).

4. J. Macek, Phys. Rev. Al, 235 (1970); J. Macek (private communication).

5. L. H. Toburen, Phys. Rev. A3, 216 (1971).

6. T. F. M. Bonsen and I. Vriens, Physica 47, 307 (1970).

7. A. Salin, J. Phys. B. 2, 631 (1969).

8. J. Macek (private communication). This Born calculation is very similar to the one described in Ref. 1.

9. G. B. Crooks and M. E. Rudd, Phys. Rev. Lett. 25, 1599 (1970).

10. L. H. Toburen, M. Y. Nakai and R. A. Langley, Phys. Rev. 171, 114 (1968). 


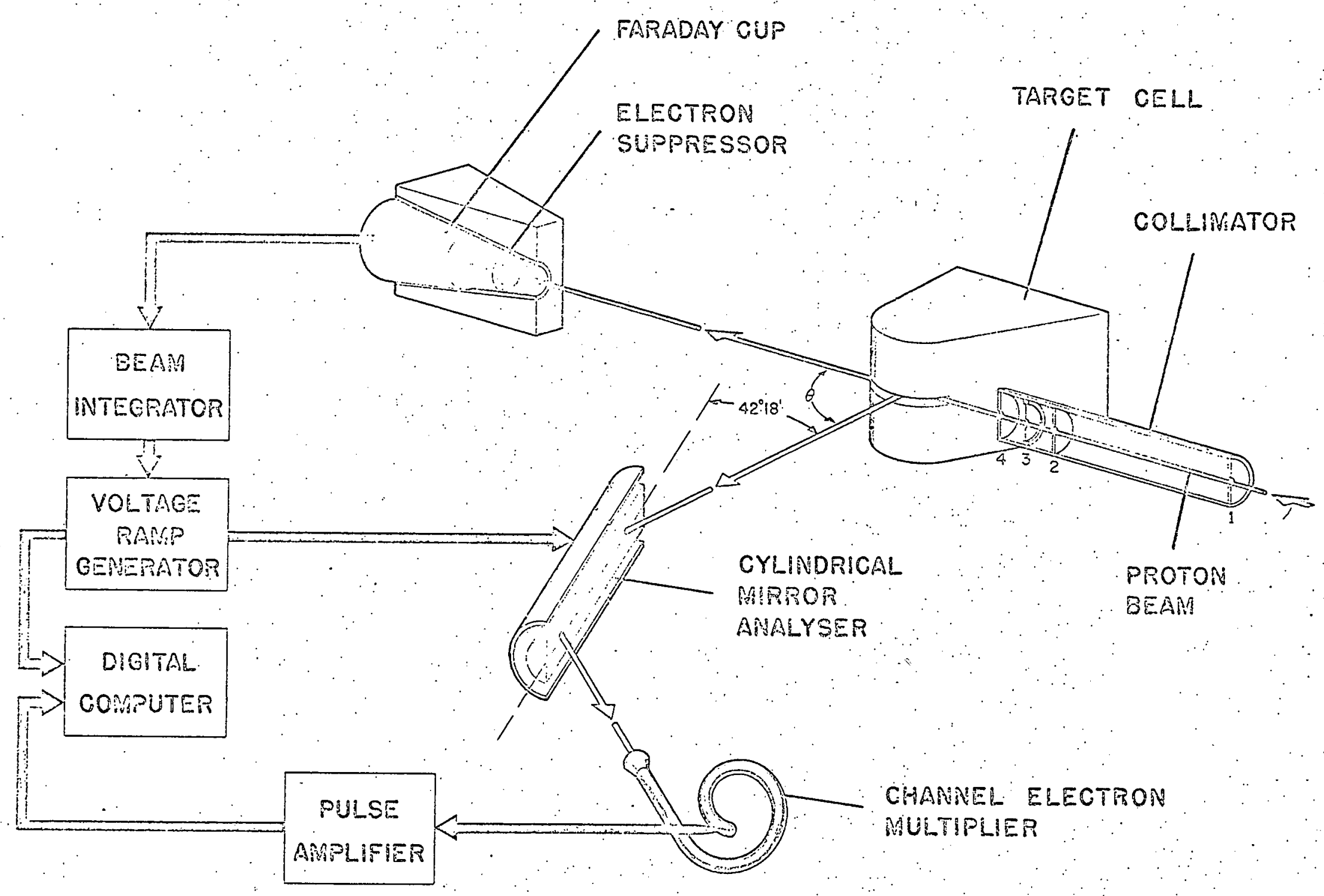




\section{2. $0 \mathrm{MeVH}^{+} \mathrm{ON} \mathrm{He}$}

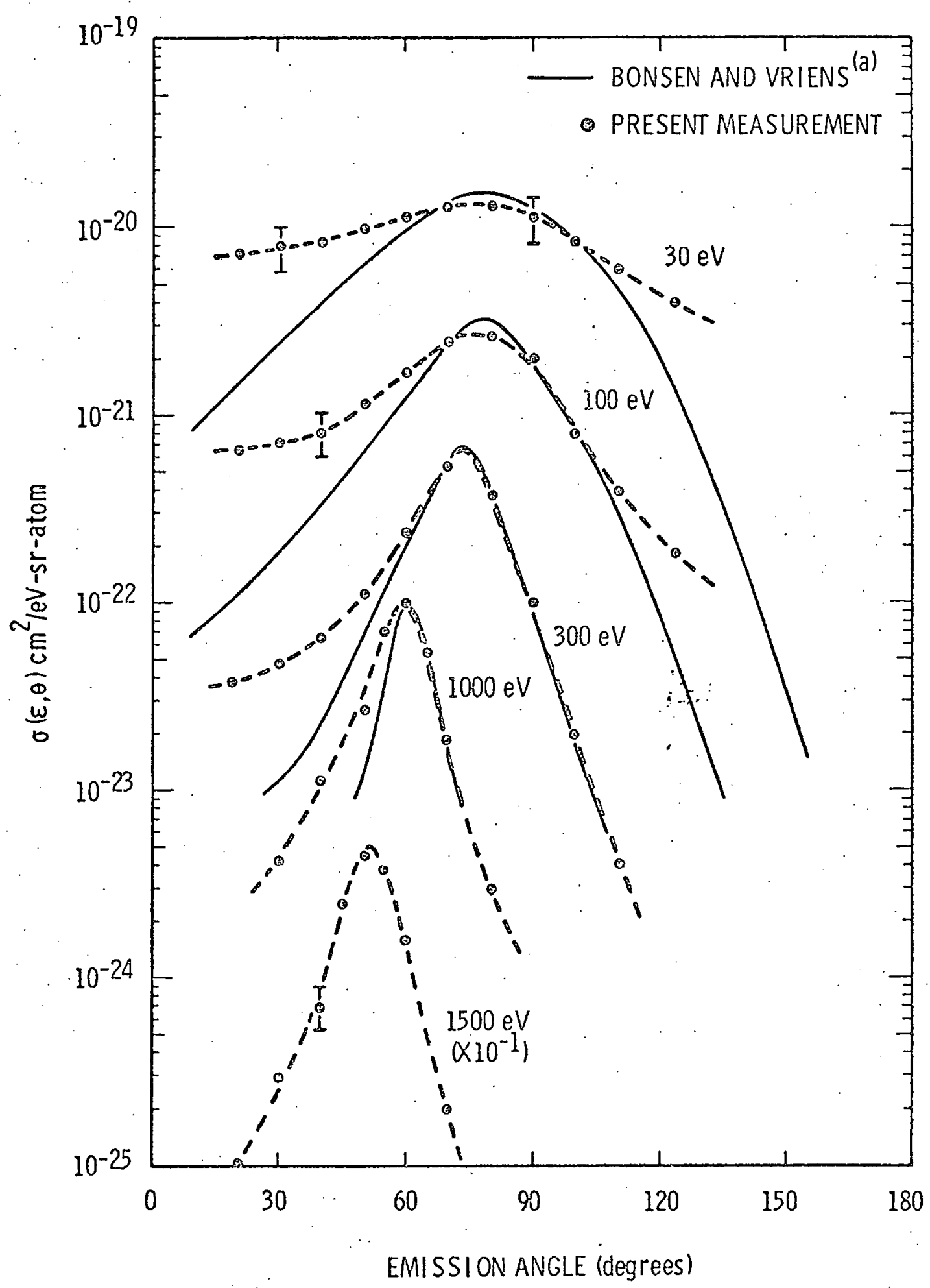

Fig. 2 


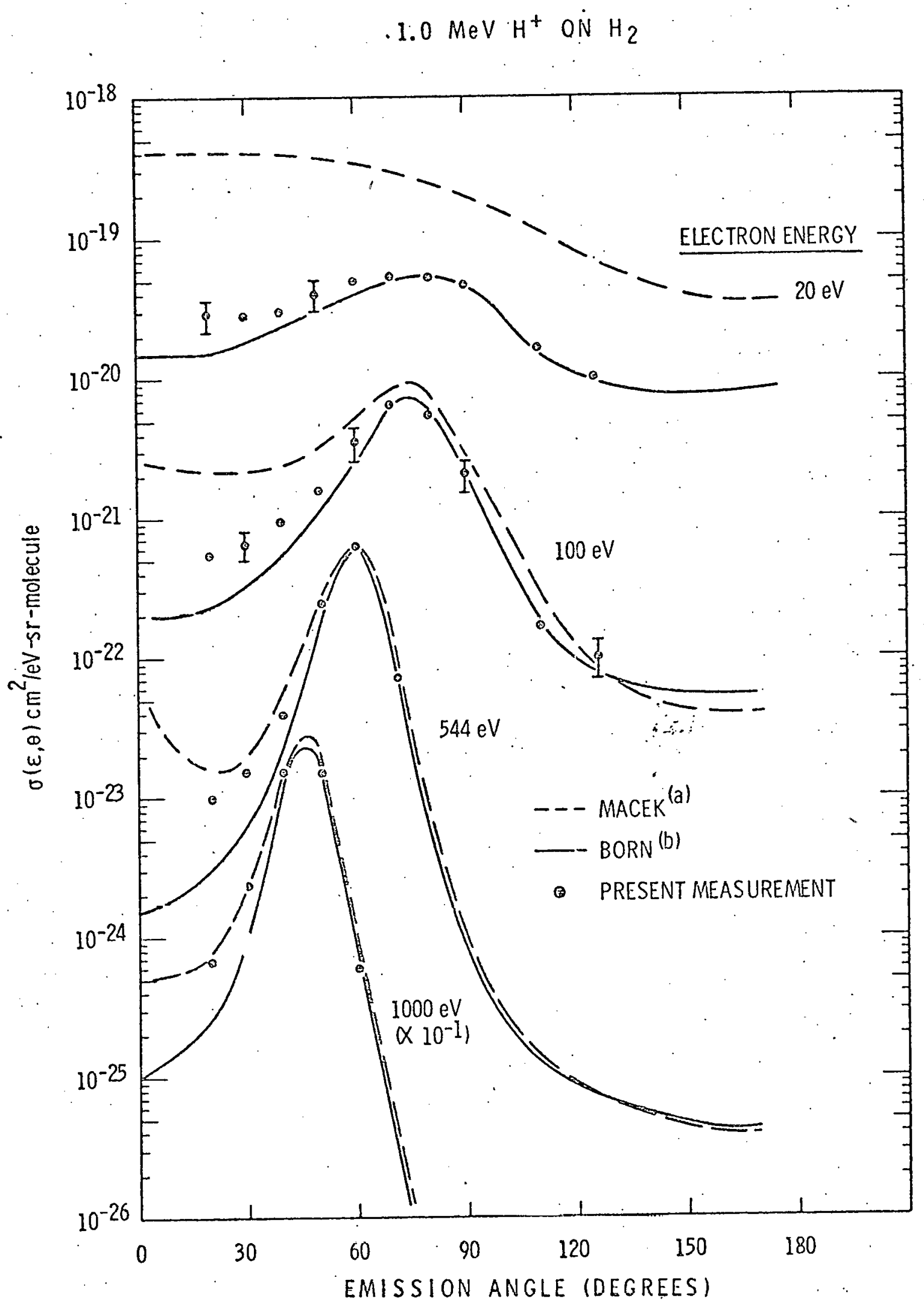

Fig. 3 


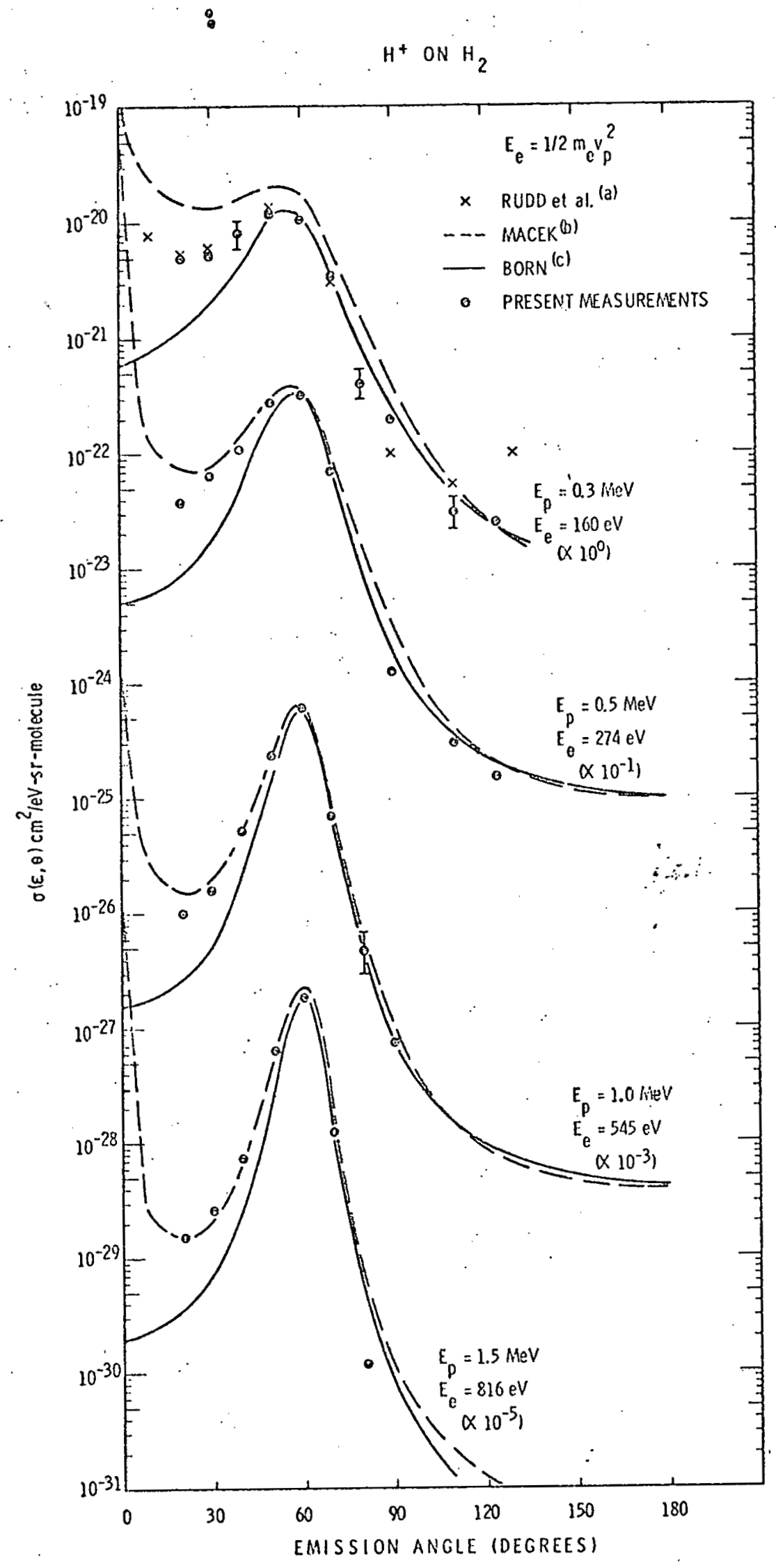

Fig. 4 


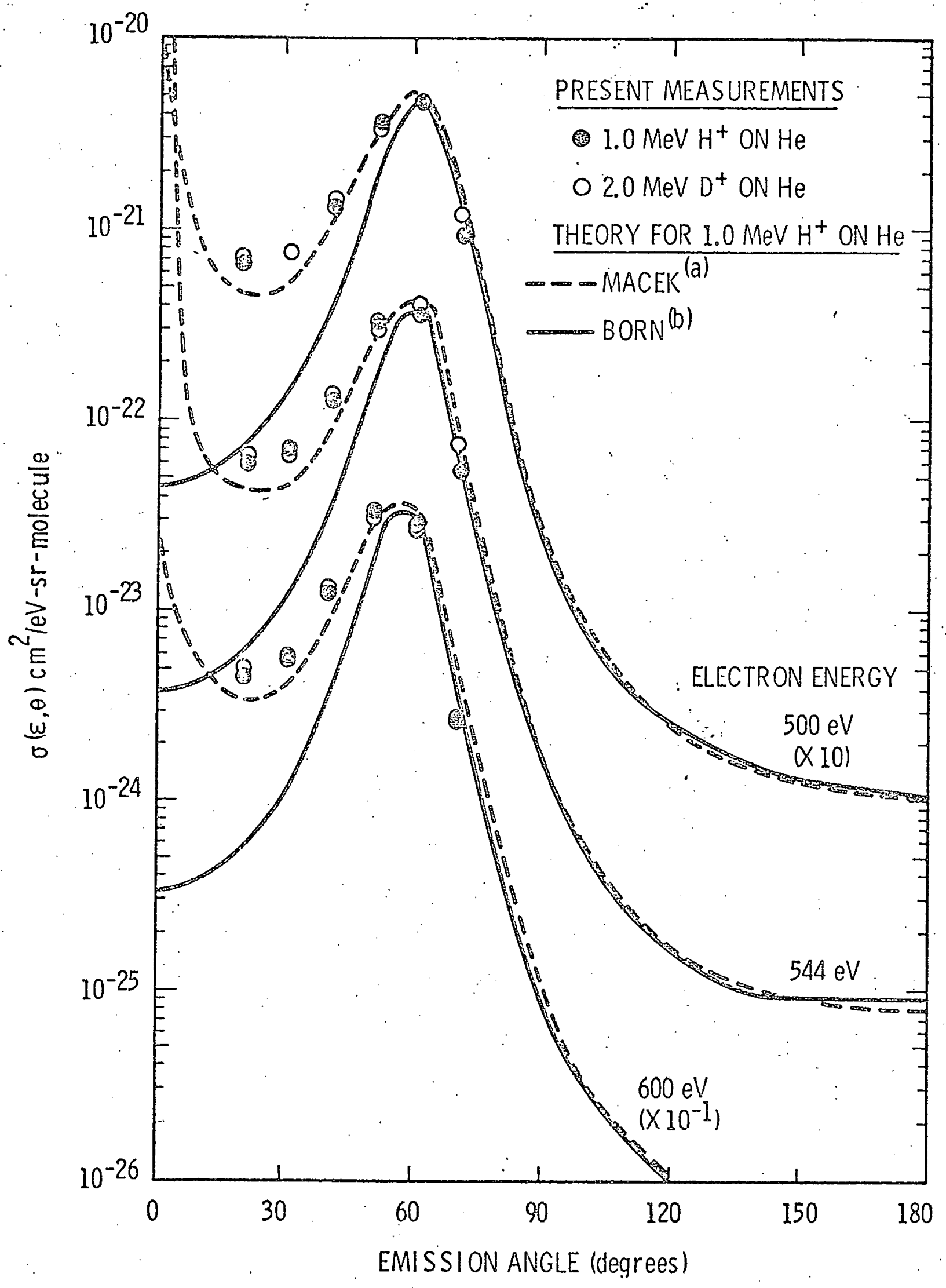

Fig. 5 


\subsection{MEV PROTON IMPACT}

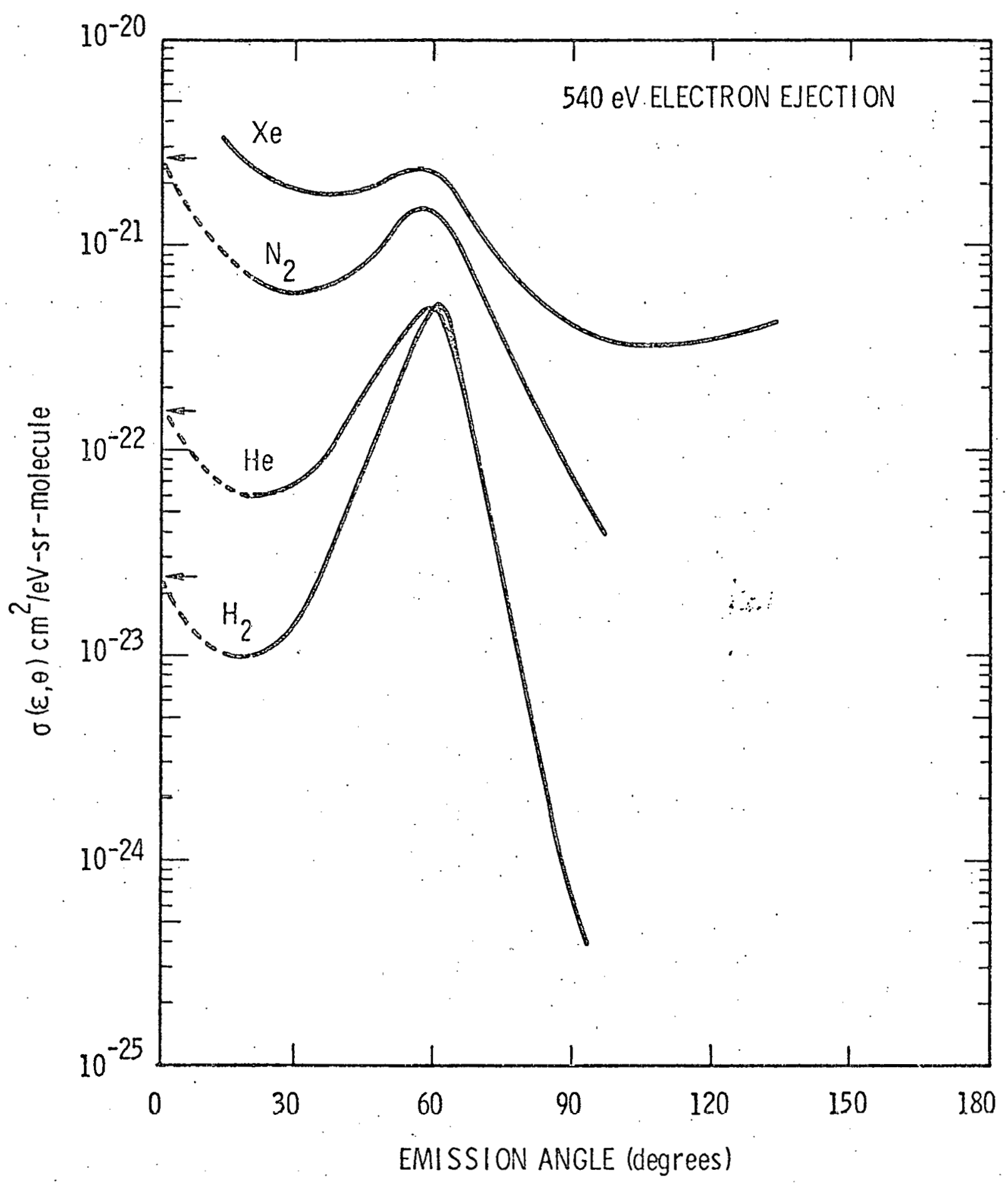

Fig: 6 